\title{
An online study of knowledge and practices of local anesthetic systemic toxicity among doctors in Sri Lanka
}

\author{
B. M. Munasinghe ${ }^{1}$, Nishanthan Subramaniam ${ }^{2} \bowtie$, Nimalan Srisothinathan ${ }^{3} \square$, \\ B. D. W. Jayamanne 4 \\ Author affiliation: \\ 1. \{ORCID:0000-0001-8373-4752\} District General hospital, Mannar, Sri Lanka. \\ 2. \{ORCID:0000-0003-4406-5896\} District General hospital, Mannar, Sri Lanka. \\ 3. \{ORCID:0000-0003-1150-9470\} District General hospital, Mannar, Sri Lanka. \\ 4. \{ORCID:0000-0002-8040-1546\} Department of Public Health, Faculty of Medicine, University of Kelaniya, Sri Lanka.
}

Correspondence: B. M. Munasinghe, Meethenwala, Walakadawaththa, Mawathagama, Sri Lanka; E-mail: malakafmp@gmail.com; Phone: +94773645827

\section{Abstract}

Background: Inadvertent over-dosage or intravascular injections may still lead to systemic toxicity. Local anesthetic systemic toxicity (LAST) could be potentially life threatening. This study focused on the current knowledge and practices in use of LA by the doctors in Sri Lanka and their ability to detect and manage an event of LAST.

Methodology: A descriptive cross-sectional study was conducted among doctors in Sri Lanka using an online selfadministered questionnaire based on AAGBI guidelines (2010). Descriptive statistics were analyzed by crosstabulations and presented as numbers and percentages using IBM-SPSS 25.

Results: The response rate was $60 \%$ out of 600 doctors. Majority were males (58\%) while $45 \%$ of the respondents were anesthetists. Ultrasound was used by $47.4 \%$ during specific LA use. The majority (74\%) considered total body weight for dose calculations. Around $50 \%$ of the respondents identified bupivacaine as the most cardiotoxic. The majority $(77 \%)$ utilized some form of monitoring and were knowledgeable on identification, prevention and initial management of LAST. Approximately 45\% identified Intralipid (ILE) as the definitive treatment of LAST, out of which, $66.8 \%$ knew the correct dose, $77.2 \%$ and $26.5 \%$, the availability and the location of the stored drug, respectively.

Conclusion: The basic knowledge about LAST was satisfactory among the respondents. A statistically significant difference on knowledge on maximum safe doses of LA, ILE in established LAST, its dosage and the availability was identified between anesthetists and non-anesthesia doctors; and postgraduate trainees and the rest of the doctors. Overall, significant lapses were noted with regard to the use of total body weight for dose calculations, use of ultrasound during LA administration and dosage, availability and storage of the definitive therapy and ILE.

Abbreviations: LA - Local anesthetics; LAST - Local anesthetic systemic toxicity; ILE - Intralipid

Key words: Local Anesthetic Systemic Toxicity; LAST, cardiac toxicity, Intralipid

Citation:_Munasinghe BM, Subramaniam N, Srisothinathan N, Jayamanne BDW. An online study of knowledge and practices of local anesthetic systemic toxicity among doctors in Sri Lanka. Anaesth. pain intensive care 2022;26(1):69-74. DOI: 10.35975/apic.v26i1.1770

Received: August 27, 2021, Reviewed: October 05, 2021, Accepted: October 10, 2021

\section{Introduction}

Local anesthetic systemic toxicity (LAST) is rare, contributed by under diagnosis and under-reporting, ${ }^{1}$ but could result in serious morbidity and mortality. ${ }^{2}$

Existing literature emphasizes the importance of knowledge on LAST. ${ }^{3}$ Knowledge and practices in recognizing LAST is essential in minimizing and ultimately managing an event of LAST. Sri Lanka has 
about 20,000 practicing doctors, and level of awareness about LAST was found to be $30 \%$ in a regional study among doctors. ${ }^{4,5}$

We reviewed the literature on factors contributing to LAST and management protocols and studied the knowledge and practices among the doctors in our study population regarding identifying, preventing and managing LAST.

\section{Methodology}

The study was conducted as a descriptive cross-sectional study among middle and intermediate-grade doctors in Sri Lanka. Considering 20,000 practicing doctors' population were eligible for our study, and level of awareness on LAST in a regional study was $30 \%$ (outcome factor of $30 \%$ selected), at $7.5 \%$ confidence limit and $95 \%$ confidence interval with a design effect (2.0) for cluster sampling, a sample size of 285 was calculated. Following attrition for $20 \%$ for nonresponders, the minimum sample size required was 342 . A self-administered questionnaire was prepared following review of literature and the Association of Anesthetists of Great Britain \& Ireland (AAGBI) guideline on LAST (2010). Face and content validity and appropriateness to culture were assessed and certified by an expert panel. A single-stage cluster sampling method was utilized. Hospitals were chosen randomly. Following establishing remote verified individual communications (via e-mail or social media, WhatsApp/Viber/ Facebook) the questionnaire was distributed. The data analysis was done with IBM SPSS (version 25) by applying relevant statistical tests. $\mathrm{P}<$ 0.05 was considered as statistically significant.

Ethical approval was obtained from the ERC of the Sri Lanka Medical Association. (ERC/20/023).

The data was collected only after completion and submission of an on-line consent form which included all the necessary instructions. No data which could lead to potential respondent identification was collected during the study.

\section{Results}

Out of 600 participants, 360 responded (response rate $=$ $60 \%)$ where $210(58.3 \%)$ were males. Median age was 32 y $(\mathrm{Q} 1=29.7, \mathrm{Q} 3=34.4, \mathrm{IQR}=4.7)$.

Nearly half had experience of 2-5 y as doctors. About $30 \%$ were postgraduate trainees from different specialties.

Distribution of responders according to subspecialty is shown in Table 1.

\subsection{Practices of using local anesthetics}

The frequency and route of usage of the three available LA; lignocaine, bupivacaine and prilocaine were studied.

\begin{tabular}{lll}
\hline $\begin{array}{l}\text { Table 1: Distribution of responders according to } \\
\text { subspecialty }\end{array}$ & Number & $\%$ \\
\hline \multicolumn{1}{|c}{ Subspecialty } & 160 & 44.4 \\
\hline Anaesthesia/ ICU & 20 & 5.6 \\
Emergency Medicine & 36 & 10.0 \\
General surgery & 39 & 10.8 \\
Medicine & 20 & 5.6 \\
Gynaecology and Obstetrics & 12 & 3.3 \\
Paediatrics & 5 & 1.4 \\
Ophthalmology & 7 & 1.9 \\
Oral and maxillofacial surgery & 6 & 1.7 \\
Orthopaedics & 7 & 1.9 \\
Radiology & 48 & 13.4 \\
Other* & $\mathbf{3 6 0}$ & $\mathbf{1 0 0}$ \\
\hline Total & & \\
\hline${ }^{*}$ ENT, plastic, urology, vascular surgery etc. & \\
\hline
\end{tabular}

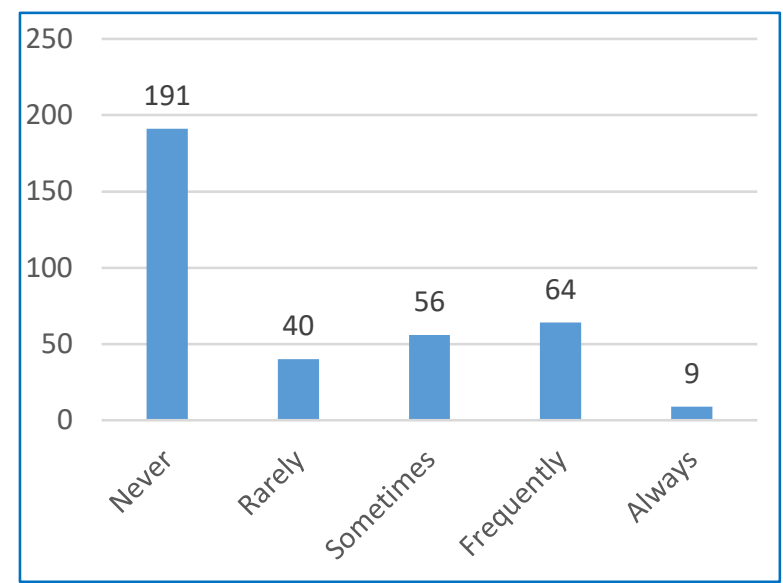

Figure 1: Frequency of the usage of ultrasound

Plain lignocaine was the most commonly used and prilocaine the least commonly used agent (Table 2).

Subcutaneous infiltration was the commonest route $(39.7 \%)$ followed by regional nerve blocks $(39.7 \%)$ and epidurals (13\%).

\subsection{Usage of ultrasound}

Most of the participants - 191 (53\%) never used ultrasound during LA administration (Figure 1).

Out of these, $38(20 \%)$ were anesthetists. Around 73 (20\%) responded as using ultrasound 'always' or 'frequently'. Majority, $63(86 \%)$ of this category were anesthetists.

The preferred mode of monitoring was found to be 'pulse oximetry' during LA ( $\mathrm{n}=234$ (65) while 120 (33.3\%) utilized pulse oximetry, ECG and non-invasive blood pressure monitoring. $14.2 \%$ (51) used at least pulse 


\begin{tabular}{lllllll}
\hline \multirow{2}{*}{ Table 2: Frequency of usage of local anesthetic agent } \\
\hline \multirow{2}{*}{ Agent } & $25(6.9)$ & $54(15)$ & $63(17.5)$ & $85(23.6)$ & $61(16.9)$ & $72(20)$ \\
\cline { 2 - 7 } & Never (\%) & $<1(\%)$ & $\mathbf{1 - 5}(\%)$ & $6-15(\%)$ & $16-30(\%)$ & $>\mathbf{3 0}(\%)$ \\
\hline Plain lignocaine & $52(14.4)$ & $77(21.4)$ & $88(24.4)$ & $71(19.7)$ & $36(10)$ & $36(10)$ \\
Lignocaine with adrenaline & $111(30.8)$ & $36(10)$ & $65(18.1)$ & $64(17.8)$ & $36(10)$ & $48(13.3)$ \\
Plain bupivacaine & $309(85.8)$ & $33(9.2)$ & $09(2.5)$ & $09(2.5)$ & 0 & 0 \\
Prilocaine & & & & & & \\
\hline
\end{tabular}

oximetry, and 86 (23.9\%) did not use any monitoring. A test dose of LA was administered by 90 (25\%) (95\% CI 20.4-29.6).

For dose calculations, the age was considered by $42.7 \%$, the comorbidities by $46.6 \%$ and ideal or lean body weight by $26.9 \%$ of the respondents. No statistical significance was identified between the anesthetists (group A) vs. non-anesthetists (group NA) $(\mathrm{P}=0.100)$, postgraduate trainees (group PG) vs. non- postgraduate (group NPG) $(\mathrm{P}=0.604)$ and doctors experienced $>10$ y (group E) vs. doctors experienced $<10$ y (group NE) $(\mathrm{P}=0.835)$.

The respondents who identified the maximum safe doses are shown in Figure 2.

Knowledge on safe doses significantly differed between PG trainees (group PG) and non-PG trainees (group NG), and anesthetists (group A) and non-anesthetist (group NA) doctors (p < 0.05) (Table 3).

A total of $172(47.8 \%)$ recognized bupivacaine as the most cardiotoxic. There was a statistically significant difference between group $\mathrm{A}$ and group NA ( $p<0.001)$ and group E vs. group NE $(\mathrm{P}=0.0003)$.

93.3\% respondents had heard about LAST and $22.5 \%$ had witnessed an episode. Approximately 95\% (95\% CI 92.7-97.3) or more had knowledge on cardiovascular $(\mathrm{n}=$ $352)$ and neurological features $(n=345)$ of LAST. Hypertension (22.5\%), and tachycardia $(45.8 \%)$ were relatively less known clinical features. Comparatively higher knowledge was elicited among group A vs. group NA, $(\mathrm{p}=$ $0.05)$ and group PG vs. group NPG $(\mathrm{P}=0.01)$.

The question about the knowledge about steps to prevent LAST revealed that $75.3 \%$ chose monitoring during LA, $78.33 \%$ aspiration and $65 \%$ ultrasound use. Around $42.5 \%$ considered addition of adrenaline to be useful. More anesthetists recognized the latter $(\mathrm{p}=0.00007)$ compared to NA group. Similar statistical significance $(\mathrm{p}=0.0005)$ was identified between PG and NPG groups.
Approximately $83 \%$ (95\% CI 79.0-86.9) respondents identified intravenous fluid and oxygen as part of the management of an established LAST while 57\% opted for prolonged cardiopulmonary resuscitation. Only $14.7 \%$ considered cardiopulmonary bypass as part of the therapy; and $15 \%$ respondents considered the use of intravenous propofol.

Intravenous ILE being the definitive therapy for LAST was recognized by 162 (45\%) (95\% CI 39.7-50.2) of the

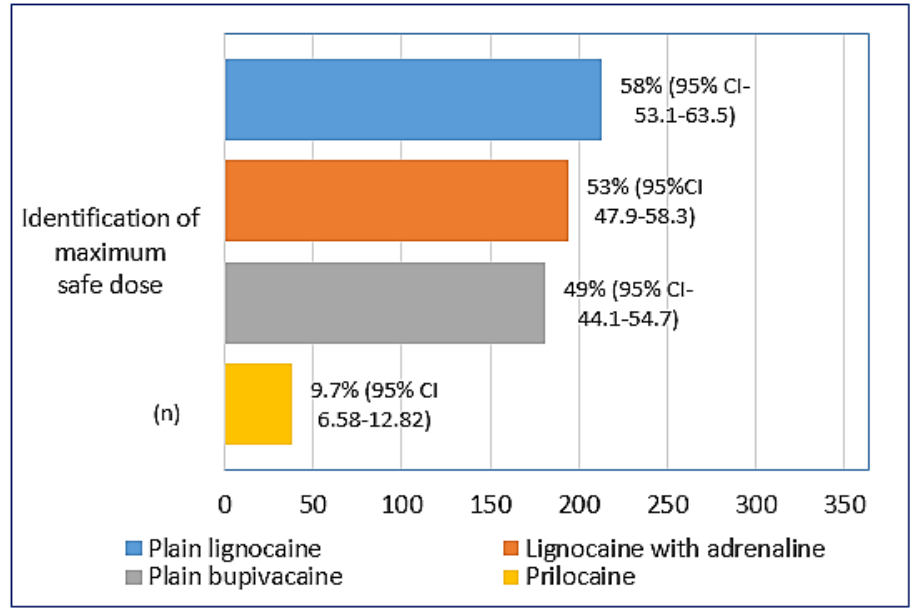

Figure 2: Identification of maximum safe doses of LA

Table 3: Correct knowledge of safe doses among PG trainees (group PG) and non PG trainees (group NG) (\%)

\begin{tabular}{|l|l|l|l|}
\hline Agent & Group PG & Group NPG & p value \\
\hline Plain lignocaine & 75.0 & 51.6 & $<0.001$ \\
\hline Lignocaine with adrenaline & 70.2 & 46.0 & $<0.001$ \\
\hline Palin bupivacaine & 67.3 & 42.0 & $<0.001$ \\
\hline Prilocaine & 17.3 & 6.6 & 0.002 \\
\hline
\end{tabular}

Table 4: Correct knowledge of safe doses among anesthetists (group A) and non-anesthetist (group NA) doctors (\%)

\begin{tabular}{|l|l|l|l|}
\hline Agent & Group A & Group NA & p value \\
\hline
\end{tabular}

\begin{tabular}{|l|l|l|l|}
\hline Plain lignocaine & 80.0 & 41.0 & $<0.001$ \\
\hline Lignocaine with adrenaline & 78.1 & 33.0 & $<0.001$ \\
\hline Palin bupivacaine & 86.8 & 19.5 & $<0.001$ \\
\hline Prilocaine & 15.0 & 5.5 & 0.003 \\
\hline
\end{tabular}


respondents; Group A $>$ NA and group PG $>$ NPG $(\mathrm{P}=$ $0.0001)$; and group $\mathrm{E}$ vs. NE $(\mathrm{P}=0.015)$ which were significant statistically. Around 66\% (95\% CI 61.0170.9) of this group identified the correct dose of the ILE; the knowledge in group A was significantly more than group NA ( $\mathrm{P}=0.001)$. Approximately $54(33.3 \%)$ of this subcategory, responded that ILE was not available in their institution. The PG respondents [108(66.6\%)] were more knowledgeable on the availability of ILE compared to NPG group ( $\mathrm{P}=0.012)$, but only $43(26.5 \%)$ of them knew about the site where it was stored.

\section{Discussion}

Local anesthetic agents are used frequently in most subspecialties of medicine. ${ }^{6}$ The growing interest in regional nerve blocks, enhanced recovery after surgery (ERAS) protocols, and multimodal analgesic regimens, have led to an increase in the usage of LA. ${ }^{7}$ Global increase in aging population may further increase the potential of LAST in the future. ${ }^{8}$

The incidence of LAST is about 0.87 per 1000 peripheral nerve blocks. ${ }^{9}$ Epidural blocks are commonly associated with LAST. ${ }^{10}$ Most cases reported are related to bupivacaine, attributed to its increased cardiotoxicity. Serious LAST can be as common as epidural hematomas and peripheral nerve injury. ${ }^{11}$ LAST could occur after continuous infusions. ${ }^{11} \mathrm{~A}$ case series revealed that about $20 \%$ cases of LAST occur outside the hospital setting, where $50 \%$ involved were non-anesthetists and $20 \%$ of events followed simple infiltration. ${ }^{11}$

Seizures, agitation and loss of consciousness are common neurological symptoms while dysarthria, perioral numbness, confusion, obtundation and dizziness are rare. Neurological symptoms typically precede cardiovascular symptoms, ${ }^{10}$ which include both tachyarrhythmias and bradyarrhythmias, which could progress to cardiac arrest. ${ }^{8}$ However, $40 \%$ of patients can present atypically where symptoms either get delayed or cardiovascular symptoms could occur without neurological manifestations. ${ }^{11}$

Our study demonstrated that, around one-quarter (24\%) did not establish any monitoring during LA administration. Monitoring electrocardiography (ECG), pulse oximetry and blood pressure are important in detecting LAST early, ${ }^{3}$ and should be continued for recurrent $^{1}$ and late-onset toxicity, especially with continuous infusions. ${ }^{12}$ The pulse oximetry was preferred in this study probably due to the ease of use.

A higher percentage $(25 \%)$ of participants stated giving a test dose. Conversely, a cross-sectional study among emergency physicians in Turkey, ${ }^{13}$ demonstrated a 5\% proportion. In another study among the ophthalmologists in Turkey, around $97.1 \%$ were not using a test dose. ${ }^{14}$ The use of a test dose is particularly useful during nerve blockade whenever critical LA volumes are used or for normal volumes in patients with co-morbidities, in view of minimizing LAST. ${ }^{15}$

Safe dose is dependent on multiple factors including the LA, the site, age, body weight, comorbidities and physiological variations such as pregnancy. ${ }^{3,8,13}$

Dose calculations based on body weight are variable in literature, where some prefer ideal body weight ${ }^{12]}$, while lean body weight is preferred by others. ${ }^{16}$ Total body weight can overestimate total dose in obese and pregnant patients, where ideal or lean body weight is more appropriate. $^{12}$ Among our respondents, only around a quarter, were using ideal or lean body weight. Comorbidities were considered by just under $50 \%$. The patients with organ dysfunction are at a higher risk of LAST, thus doses should be titrated. ${ }^{11}$

Considering the ubiquitous use of LA except for prilocaine, a significant lapse of knowledge was noted with regard to maximum safe doses. Just under half identified bupivacaine as the most cardiotoxic. Seven respondents considered intravenous use of bupivacaine to be catastrophic and to be avoided at all times.

Airway management, oxygenation, ventilation and control of seizures are essential components of supportive management in established LAST. ${ }^{3,8}$ Intravenous $20 \%$ lipid emulsion (ILE) given as a 1.5 $\mathrm{ml} / \mathrm{kg}$ bolus, followed by an infusion of $15 \mathrm{ml} / \mathrm{kg} / \mathrm{h}$ is used as the specific management. ILE and management protocols should be readily available where LA is utilized. ${ }^{1,8}$

As steps in preventing LAST, monitoring, aspiration and use of ultrasound were considered by most. Addition of adrenaline to LA was the least preferred. The physiological response to adrenaline (increments of heart rate by around 10 beats/min and blood pressure by $10-15$ $\mathrm{mmHg}$ ), could be an important marker of intravascular injection, as aspiration could be falsely negative in around 2\%. ${ }^{11}$ An overall comparative lack of knowledge on the prodromal features of LAST was evident. Given that the typical pattern of toxicity may not be seen among $40 \%$, the detection of prodromal features could be decisive.

Ultrasound minimizes LAST by real-time visualization and use of lower volumes with evidence of $65 \%$ reduction compared to nerve stimulation alone. ${ }^{9}$ Even though, the majority in our study considered ultrasound, its practical use was far less. This could be explained by the preference, access to ultrasound and routes of LA administered. For instance, nearly $90 \%$ who utilized ultrasound 'Always' or 'Frequently' were anesthetists, who perform more regional nerve blocks ultrasonically.

The basic management of an established LAST was known by a significantly higher number of respondents 
with preference for fluids, oxygen, seizure management and prolonged cardiopulmonary resuscitation.

The definitive therapy, ILE was relatively less known by the respondents while few chose propofol. ILE should not be substituted by propofol due to relatively low lipid content, potential cardiovascular compromise and need of larger volumes. ${ }^{17}$

A study by Edwards et al., demonstrated an overall deficit in knowledge on LAST (including ILE therapy) in a Maternity unit in a UK Hospital. However, teaching programs led to a significant improvement in knowledge. $^{18}$ A Danish study conducted among anesthetists, revealed that about 50\% knew about lipid therapy but were not aware how to acquire ILE. ${ }^{19}$ In a cross-sectional study in Turkey, $42 \%$ of the emergency physicians identified ILE dosage. ${ }^{13}$

The AAGBI and AHA (American Heart Association) have endorsed the use of ILE in LAST, it being an emergency where minutes count, and quick access to ILE is undoubtedly decisive.

A significant statistical difference in knowledge was found between postgraduate trainees vs. nonpostgraduate trainees, anesthetists vs. non-anesthetist doctors and experienced (>10 y) vs. less experienced doctors, on maximum safe doses, cardiotoxicity of bupivacaine, prodromal cardiovascular features and addition of adrenaline and performance of a test dose. ILE as the drug of choice, dosage, availability and place of storage were known with significant statistical significance by more anesthetists and postgraduate trainees. The familiarity, increased frequency of LA use, ILE mainly stored in operating theatres and continuous medical education possibly explain this observation.

\section{Limitations}

The response rate was relatively low for the study. Fields where topical LA is used in higher quantities (respiratory medicine for bronchoscopic procedures for instance), the same pattern was noticed. The correct answers were provided to the respondents after the study; however, the prevailing pandemic hindered the completion of the audit cycle, which could have been more informative.

\section{Conclusion}

The basic knowledge on LAST was satisfactory among the respondents. Significant lapses were identified with regard to use of the total body weight for dose calculations, use of ultrasound during LA administration and dosage and importantly availability and storage of the definitive therapy - ILE.

\section{Recommendations}

The authors suggest the following:
- Continuing medical education programs on LAST to be conducted particularly for non-anesthetic doctors, doctors who are not engaged in postgraduate studies and who have completed specific length of service (e.g. 5 y)

- Inclusion of LAST to the curriculum of the nonanesthetic postgraduate trainees.

- LAST management protocols to be displayed at locations of LA use. (main operating rooms, emergency operating rooms, radiology and dental suites, and any other place of high-volume LA use).

- ILE to be available at places where LA is used/ daily checklists/ stocks to be displayed.

- Prompt reporting and auditing of adverse events related to $\mathrm{LA}$.

\section{Acknowledgement}

The authors would like to pay their gratitude to all the respondents, Dr. M.D.C.J.P. Jayamanne and Dr. K.A. Jayasundara (consultant pediatricians, District General Hospital, Mannar, Sri Lanka) for their valuable insights.

\section{Conflict of interest}

No conflicts of interest were declared by the authors. Study was self-funded by the authors No external funding was received.

\section{Authors' contribution}

BM conceived the concept for the study. BM, SN, NS did the literature review. BM, SN, NS and WJ designed the study. BM, NS and SN developed the proposal and proceeded to ethical approval. BM, SN, NS collected data. All authors were involved in data analysis, compiling, and reviewing the final manuscript.

\section{References}

1. Weinberg G. ESRA19-0705 Systemic local anaesthetic toxicity: last and beyond. BMJ Publishing Group Ltd; 2019.

2. Gitman M, Fettiplace MR, Weinberg GL, Neal JM, Barrington MJ. Local anesthetic systemic toxicity: A narrative literature review and clinical update on prevention, diagnosis, and management. Plast Reconstr Surg. 2019;144(3):783-95. [PubMed] DOI: 10.1097/PRS.0000000000005989

3. Barrington MJ, Weinberg GL, Neal JM. A call to all readers: educating all surgeons on preventing and treatment of local anaesthetic systemic toxicity. ANZ J Surg. 2016;86(9):636-7. [PubMed] DOI: 10.1111/ans.13667

4. De Silva D. How many doctors should we train for Sri Lanka? System dynamics modelling for training needs. Ceylon Med J. 2017;62(4):233-7. [PubMed] DOI: 10.4038/cmj.v62i4.8573

5. Sagir A, Goyal R. An assessment of the awareness of local anesthetic systemic toxicity among multi-specialty 
postgraduate residents. J Anesth. 2015;29(2):299-302. [PubMed] DOI: 10.1007/s00540-014-1904-9

6. Vasques F, Behr AU, Weinberg G, Ori C, Di Gregorio G. A review of local anesthetic systemic toxicity cases since publication of the American Society of Regional Anesthesia Recommendations: to whom it may concern. Reg Anesth Pain Med. 2015;40(6):698-705. [PubMed] DOI: 10.1097/AAP.0000000000000320

7. Weibel S, Jokinen J, Pace N, Schnabel A, Hollmann M, Hahnenkamp K, et al. Efficacy and safety of intravenous lidocaine for postoperative analgesia and recovery after surgery: a systematic review with trial sequential analysis. $\mathrm{Br} \mathrm{J}$ Anaesth. 2016;116(6):770-83. [PubMed] DOI: 10.1093/bja/aew101

8. Guideline AS. Management of severe local anesthetic toxicity. London: The Association of Anaesthetists of Great Britain \& Ireland. 2010. DOI: 10.21466/g.MOSLAT2.2010

9. Barrington MJ, Kluger R. Ultrasound guidance reduces the risk of local anesthetic systemic toxicity following peripheral nerve blockade. Reg Anesth Pain Med. 2013;38(4):289-99. [PubMed] DOI: 10.1097/AAP.0b013e318292669b

10. Di Gregorio G, Neal JM, Rosenquist RW, Weinberg GL. Clinical presentation of local anesthetic systemic toxicity: a review of published cases, 1979 to 2009. Reg Anesth Pain Med. 2010;35(2):181-7--7. [PubMed] DOI: 10.1097/aap.0b013e3181d2310b

11. Neal JM, Barrington MJ, Fettiplace MR, Gitman M, Memtsoudis SG, Mörwald EE, et al. The third American Society of Regional Anesthesia and Pain Medicine practice advisory on local anesthetic systemic toxicity: executive summary 2017. Reg
Anesth Pain Med. 2018:43(2):113-23. [PubMed] DOI: 10.1097/AAP.0000000000000720

12. Christie LE, Picard J, Weinberg GL. Local anaesthetic systemic toxicity. BJA Edu. 2015;15(3):136-42. DOI: 10.1093/bjaceaccp/mku027

13. ILHAN B, Demir MC. Knowledge of local anesthetic systemic toxicity among emergency medicine physicians: a crosssectional study. Signa Vitae. 2020;16(2):148-54. DOI: 10.22514/sv.2020.16.0040

14. 1Urfalıoğlu A, Urfalığlu S, Öksüz G. The knowledge of eye physicians on local anesthetic toxicity and intravenous lipid treatment: Questionnaire study. Turk J Ophthalmol. 2017;47(6):320. [PubMed] DOI: 10.4274/tjo.79446

15. Galindo Gualdrón LA. Test dose in regional anesthesia. Revista Colombiana de Anestesiología. 2014;42(1):47-52. DOI: 10.1016/j.rcae.2013.11.002

16. El-Boghdadly K, Pawa A, Chin KJ. Local anesthetic systemic toxicity: current perspectives. Local Reg Anesth. 2018;11:35. [PubMed] DOI: 10.2147/LRA.S154512

17. Beecroft C, Davies G. Systemic toxic effects of local anaesthetics. Anaesth Intensive Care Med. 2010;11(3):98-100.

18. Edwards AE, Bowsher GM, Deepak S, Ali M. Improving local anaesthetic systemic toxicity (LAST) awareness in maternity care using tailored educational tools. BMJ Open Qual. 2018;7(2). [PubMed] DOI: 10.1136/bmjoq-2017-000070

19. Jensen-Gadegaard P, Skjønnemand M, Damgaard-Jensen J, Gottschau B. Limited knowledge of lipid rescue therapy in local anaesthetic systemic toxicity. Dan Med Bull. 2011;58(1):A4226. [PubMed] 